\title{
QUANTUM SYSTEMS \\ IN REGULAR AND STOCHASTIC FIELDS. CREATION AND DESTRUCTION OF THE COHERENCE
}

\author{
A. V. Gorokhov ${ }^{1}$
}

Department of General and Theoretical Physics, Samara State University, Samara, Russia

The problem of the coherent state generation with definite parameters for multilevel quantum systems is investigated. The interaction with external environment and stochastic fields can destroy the coherence. The competition of these processes is considered on the basis of Fokker-Planck equations approach, derived from master equation for the density matrix of the system. Examples of the coherent states dynamics for two-level atoms in an external stochastic field in a nonideal resonator are considered. Average over the realizations of stochastic fields is performed for the case of white Gaussian noise and Kubo-Anderson process. Explicit formulas for probability and shape of radiation line are obtained.

Исследована проблема генерации когерентных состояний для многоуровневых квантовых систем. Взаимодействие с внешним окружением и стохастическими внешними полями разрушает когерентность. Конкуренция этих процессов изучена на основе подхода уравнений Фоккера-Планка, выведенных из кинетического уравнения для матрицы плотности системы. Рассмотрен пример динамики когерентных состояний для двухуровневых атомов во внешнем стохастическом поле в неидеальном резонаторе. Выполнено усреднение по реализациям стохастического поля в случаях белого гауссова шума и процесса Кубо-Андерсона. Выведены явные формулы для вероятностей найти атом на верхнем и нижнем уровне и контура линии излучения.

\section{INTRODUCTION}

Coherent states (CS) approach is a very important and powerful tool in modern theoretical physics [1-3]. CS found many interesting applications in quantum optics, atomic and molecular physics, condensed matter physics, quantum field theory and quantum calculations and cryptography.

The purpose of this paper consists in considering the coherent state generation problem for atomic systems and destroying their coherency by influence of large dissipative environment and interaction with external stochastic fields.

\footnotetext{
${ }^{1}$ E-mail: gorokhov@ssu.samara.ru
} 


\section{GENERATION OF ATOMIC COHERENT STATES}

It is well known that single $n$-level atom (or cooperative system of such atoms) in external homogeneous classical electromagnetic field has a dynamical symmetry of the group $S U(n)$, see, for example, the book [2]. For the case of full symmetric irreducible representation $T(g) \equiv D(p, 0, \ldots, 0)$ of $S U(n) \mathrm{CS}$ is given by the formula

$$
|z\rangle=\left(1+\sum_{\alpha=1}^{n-1} z^{\alpha} \bar{z}^{\alpha}\right)^{-p} \prod_{\beta=1}^{n-1} \exp \left(z^{\beta} \hat{E}_{\beta}^{+}\right)|0\rangle,
$$

where $z=\left(z^{1}, \ldots, z^{n-1}\right) \in S U(n) / U(n-1) \approx C P^{n-1}$ and $|0\rangle$ - the vector of dominant weight of the representation and operators $\hat{E}_{1}^{+}, \ldots, \hat{E}_{n-1}^{+}$are the raising Cartan-Weyl operators that do not belong to the family of $U(n-1)$ subgroup generators.

System of equations for the CS parameters has a form of generalized Rikkati equations [4]

$$
i \dot{z}^{\alpha}=H_{\alpha n}(t)+\sum_{\beta=0}^{n-1}\left[H_{\alpha \beta}(t)-H_{n n}(t) \delta_{\alpha \beta}\right] z^{\beta}-\sum_{\beta=0}^{n-1} H_{n \beta}(t) z^{\alpha} z^{\beta},
$$

where $\left(H_{n l}(t)\right)=H(t)$ is $n \times n$ Hermitian matrix of the Hamiltonian with zero trace.

These equations follow from the time-dependent Schrödinger equation, if one presents the state vector $|\Psi(t)\rangle$ in the form $|\Psi(t)\rangle=\mathrm{e}^{i \phi(t)}|z(t)\rangle$, where $|z(t)\rangle$ is CS of group $S U(n)$ which is the dynamical group of the system. The special feature of Eqs. (2) lies in the fact that they have the same form for all full symmetric irreducible representations of $S U(n)$.

A solution of the Cauchy problem for Eqs. (2) takes a form of the multilinear-fractional function mapping of initial point $z\left(t_{0}\right)=\left(z^{1}\left(t_{0}\right), \ldots, z^{n-1}\left(t_{0}\right)\right) \in S U(n) / U(n-1)$ with $S U(n)$ group action. Substituting $z^{\alpha}=\eta^{\alpha} / \eta^{n}, \alpha=1, \ldots, n-1$ into Eqs. (2), we get the system of linear differential equations

$$
i \hbar \dot{\eta}^{k}=\sum_{l=1} H_{k l}(t) \eta^{l}, \quad k=1, \ldots, n .
$$

The solution of Eqs. (3) is determined by the action of the unitary matrix $\theta\left(t, t_{0}\right) \in S U(n)$ on initial $n$-dimensional vector $\left(\eta^{1}\left(t_{0}\right), \ldots, \eta^{n}\left(t_{0}\right)\right)$, and takes the form

$$
\eta^{k}(t)=\sum_{l=1}^{n}\left(\theta\left(t, t_{0}\right)\right)_{k l} \eta^{l}\left(t_{0}\right)
$$

As a result, we get

$$
z^{\alpha}(t)=\frac{\sum_{\beta=1}^{n-1} \theta_{\alpha \beta}\left(t, t_{0}\right) z^{\beta}\left(t_{0}\right)+\theta_{n \alpha}\left(t, t_{0}\right)}{\sum_{\beta=1}^{n-1} \theta_{n \beta}\left(t, t_{0}\right) z^{\beta}\left(t_{0}\right)+\theta_{n n}\left(t, t_{0}\right)} .
$$

The CS dynamics for two-level atoms, as well as in the case of atoms with $2 j+1$ equidistant levels, is governed by the Rikkati equation

$$
i \dot{z}=A(t)+\omega_{0} z-\bar{A}(t) z^{2} .
$$


For the case of linear polarized monochromatic electromagnetic field $A(t)=A \exp (-i \omega t)$, $\omega$ is the field frequency, the constant $A$ is proportional to product of electric field strength and atomic dipole momentum transition; $\omega_{0}$ is atomic transition frequency.
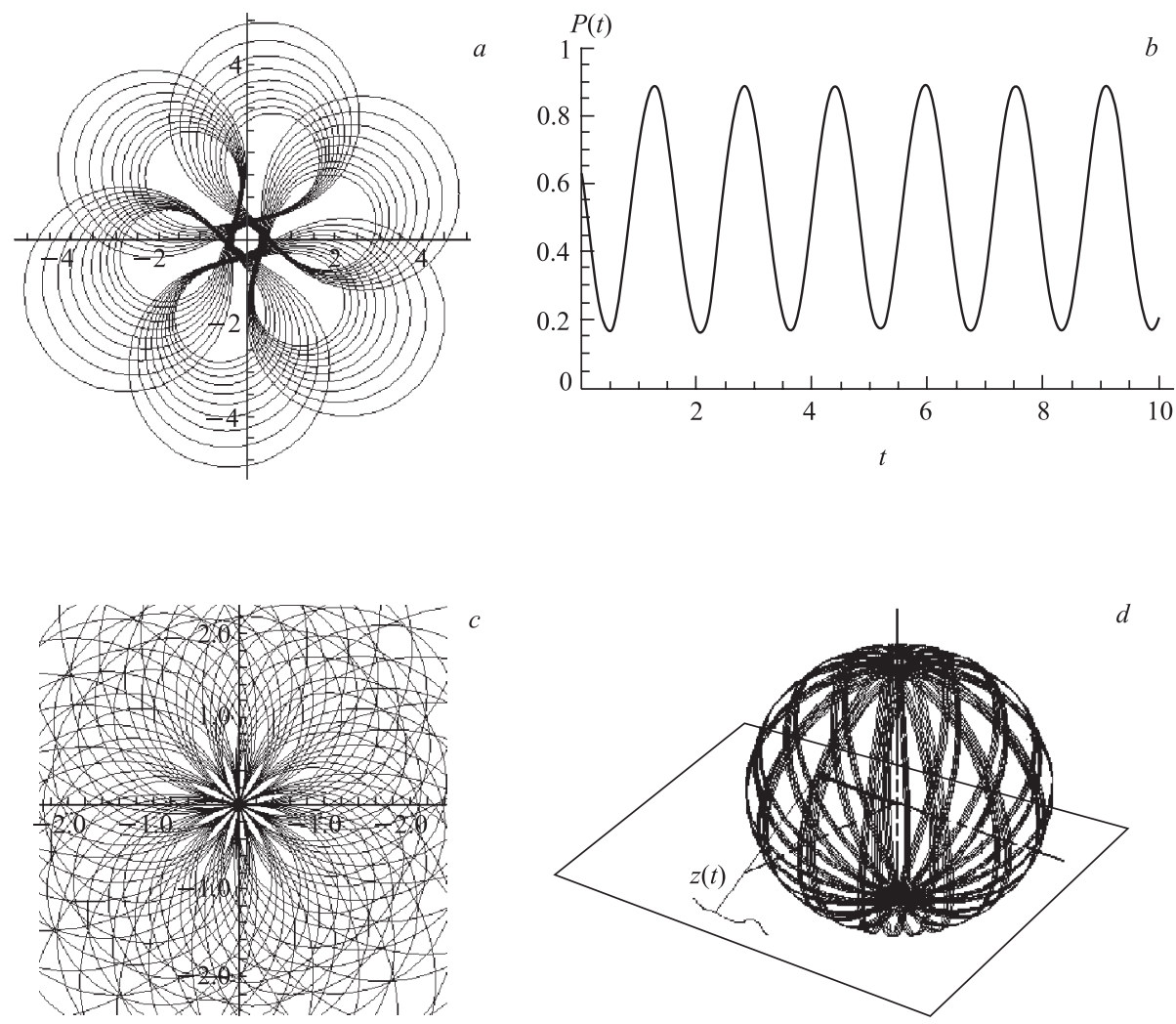

Fig. 1. Coherent state dynamics for two-level atom: $a, b$ ) trajectories on the complex plane $z=x+i y$ and probability $P(t)$ to find atom on upper level for the case $z(0)=1+i, \omega_{0}=1, \omega=2 / 3, A=2$; $c, d)$ trajectories in the the bounded complex plane region: $-2<\operatorname{Re} z, \operatorname{Im} z<2$ and Bloch sphere map of the entire trajectory for the case $z(0)=1+i, \omega_{0}=1, \omega=1.333, A=10$, respectively. The calculation time is limited by the interval $t \in[0,200]$

The CS dynamics may be visualized by the motion of the point on the complex plane or on the Bloch sphere $S_{2}$. This is shown in Fig. 1. We have used in our calculations dimensionless units (frequency $\omega_{0}=1$ ). It is evident that trajectories of CS have some remarkable «symmetry», but the atom excitation probability, determined by the simple Rabi formula

$$
P(t)=\frac{A^{2} \sin ^{2} \Omega t}{\left(\omega-\omega_{0}\right)^{2}+A^{2}}
$$

(and valid, of course, if $z(0)=0$ ), does not depend on fine details of internal CS dynamics. Here $\Omega=(1 / 2) \sqrt{\left(\omega-\omega_{0}\right)^{2}+A^{2}}$ is the well-known Rabi frequency. 
Figure 2 shows an example of CS generation for the two-level atom by influence of the Gaussian pulse $A(t)=A \exp \left[-i \omega t-\left(t-t_{0}\right)^{2} / \tau^{2}\right]$, here $\tau$ is the duration time of the pulse.
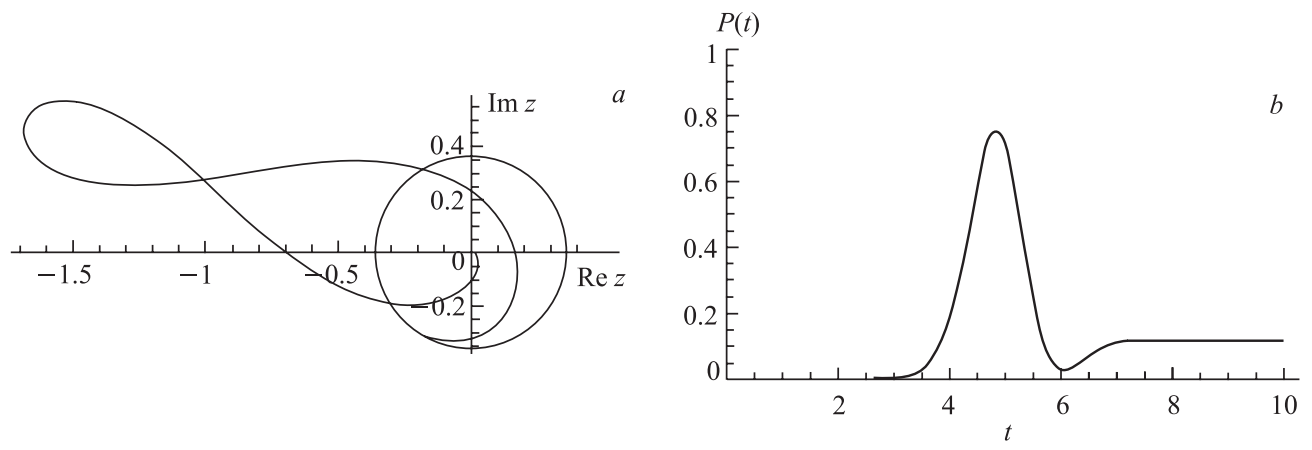

Fig. 2. Coherent state generation for the two-level atom: $a$ ) the trajectory on the complex plane $\left.z=x+i y,\left|z_{0}\right| \approx 0.358 ; b\right)$ the probability $P(t)$ to detect atom on the upper level $\left(z(0)=0, \omega_{0}=1\right.$, $\left.\omega=2, A=1.5, \tau=\sqrt{3 / 5}, t_{0}=5\right)$

In particular, the initially nonexcited atom after short pulse influence goes to any CS $\left|z_{0} \mathrm{e}^{-i \omega_{0} t}\right\rangle$ and the point $z(t)$ should break into rotation with frequency $\omega_{0}$ in a circle of radius $\left|z_{0}\right|$. In contrast with the previous case, the time dependence of the probability $P(t)$ does not follow the simple formula (5). When $t-t_{0} \sim \tau$, the probability $P(t) \approx 1$, and if the pulse action is finished, the probability tends to any constant determined by the formula

$$
P(t) \rightarrow \frac{\left|z_{0}\right|^{2}}{1+\left|z_{0}\right|^{2}}, \quad t \gg \tau .
$$

Changing the pulse parameters, we may get, in principle, different atomic CS with wanted characteristics.

Similar results are also true for atom (atoms) with $n$ levels of energy. For example, for the three-level atom we need CS

$$
\left|z_{1}, z_{2}\right\rangle=\left(1+z_{1} \bar{z}_{1}+z_{2} \bar{z}_{2}\right)^{-\mu_{1}+3 / 2 \mu_{2}} \mathrm{e}^{z_{1} \hat{L}_{+}} \mathrm{e}^{z_{2} \hat{J}_{+}}|0\rangle,
$$

where $\left(z_{1}, z_{2}\right) \in S U(3) / U(2) \approx C P_{2}$, the numbers $\mu_{1}$ and $\mu_{2}$ are eigenvalues of two diagonal operators in Cartan-Weyl basis of $S U(3)$ Lie algebra, and $\hat{L}_{+}$and $\hat{J}_{+}$are raising operators not belonging to the stationary subalgebra $U(2)$ of the atomic «vacuum» state $|0\rangle$.

\section{QUANTUM SYSTEM IN STOCHASTIC FIELD AND NONIDEAL CAVITY}

In this section we briefly consider two-level atom in nonideal cavity (heat bath) and with external fields $\Omega(t)$ and $\xi(t)$. The interaction with stochastic field may be described with the Hamiltonian [5]

$$
\hat{H}_{\mathrm{st}}=\hbar\left(\Omega(t) \hat{J}_{3}+\xi(t) \hat{J}_{+}+\bar{\xi}(t) \hat{J}_{-}\right)
$$


where $\Omega(t)$ determines a random shift of the atomic levels, $\xi(t), \bar{\xi}(t)$ are random functions which are proportional to the strength of stochastic field and should produce transitions between atomic levels:

$$
\begin{array}{r}
\frac{\partial \hat{\rho}}{\partial t}=\frac{\gamma}{2}\left[(\langle\nu\rangle+1)\left(2 \hat{J}_{-} \hat{\rho} \hat{J}_{+}-\hat{J}_{+} \hat{J}_{-} \hat{\rho}-\hat{\rho} \hat{J}_{+} \hat{J}_{-}\right)+\langle\nu\rangle\left(2 \hat{J}_{+} \hat{\rho} \hat{J}_{-}-\hat{J}_{-} \hat{J}_{+} \hat{\rho}-\hat{\rho} \hat{J}_{-} \hat{J}_{+}\right)\right]+ \\
+K_{\Omega}(t)\left(2 \hat{J}_{3} \hat{\rho} \hat{J}_{3}-\hat{J}_{3}^{2} \hat{\rho}-\hat{\rho} \hat{J}_{3}^{2}\right)+K_{\xi}(t)\left(2 \hat{J}_{+} \hat{\rho} \hat{J}_{-}+\hat{J}_{-} \hat{\rho} \hat{J}_{+}-\hat{\rho} \hat{J}_{-} \hat{J}_{+}-\hat{J}_{+} \hat{J}_{-} \hat{\rho}\right)+ \\
+\bar{K}_{\xi}(t)\left(2 \hat{J}_{+} \hat{\rho} \hat{J}_{-}+\hat{J}_{-} \hat{\rho} \hat{J}_{+}-\hat{\rho} \hat{J}_{+} \hat{J}_{-}-\hat{J}_{-} \hat{J}_{+} \hat{\rho}\right), \quad(8)
\end{array}
$$

where $\gamma$ is the damping constant; $\langle\nu\rangle$ is heat bath mean number of quanta,

$$
K_{\Omega}(t)=\int_{0}^{t}\left\langle\Omega(t) \Omega\left(t_{1}\right)\right\rangle d t_{1}, \quad K_{\xi}(t)=\int_{0}^{t}\left\langle\xi(t) \bar{\xi}\left(t_{1}\right)\right\rangle \mathrm{e}^{-\omega_{0}\left(t_{1}-t\right)} d t_{1} .
$$

This master equation is exact, if we restrict our consideration to the information included in two-time stochastic fields correlators $\left\langle\Omega(t) \Omega\left(t_{1}\right)\right\rangle$ and $\left\langle\xi(t) \bar{\xi}\left(t_{1}\right)\right\rangle$. We suppose that stochastic fields are the stationary processes with known type of statistics [7].

Presenting the density matrix in diagonal form $\hat{\rho}(t)=\int_{\mathcal{X}} d \mu(z, \bar{z}) \mathcal{P}(z, \bar{z}, t)|z\rangle\langle z|$, where $|z\rangle-S U(2) \mathrm{CS}$, one may reduce the operator master equation to the Fokker-Planck equation for density matrix symbol $\mathcal{P}$. Omitting some details, we present here the expression of the radiation line contour, calculated for the case of Kubo-Anderson random processes:

$$
\begin{aligned}
& g(\omega)=\int_{0}^{t} d t \mathrm{e}^{-i \omega_{0} t}\left\langle\hat{J}_{+}(t) \hat{J}_{-}(0)\right\rangle= \\
& \quad=\frac{1}{\pi} \frac{\eta+\delta+\frac{\sigma_{\Omega}^{2}}{\nu_{\Omega}}+2 \sigma_{\xi}^{2} \frac{\nu_{\xi}}{\nu_{\xi}^{2}+\omega_{0}^{2}}}{\left(\omega-\omega_{0}-2 \sigma_{\xi}^{2} \frac{\omega_{0}}{\nu_{\xi}^{2}+\omega_{0}^{2}}\right)^{2}+\left(\eta+\delta+\frac{\sigma_{\Omega}^{2}}{\nu_{\Omega}}+2 \sigma_{\xi}^{2} \frac{\nu_{\xi}}{\nu_{\xi}^{2}+\omega_{0}^{2}}\right)^{2}},
\end{aligned}
$$

where $\delta=(\gamma / 2)\langle\nu\rangle, \eta=\gamma / 2)(\langle\nu\rangle+1) ; \sigma_{\Omega}^{2}, \sigma_{\xi}^{2}$ and $\nu_{\Omega}, \nu_{\xi}$ are dispersions and frequencies of random processes $\Omega$ and $\xi$, respectively.

The calculated time dependences of the probabilities $W_{2}, W_{1}$ to find atom in the upper and lower states, respectively, are the following:

$$
\begin{aligned}
& W_{2}(t)=\frac{1}{2}-\frac{1}{2} \frac{\eta-\delta}{\eta+\delta}\left(1-\mathrm{e}^{-2(\eta-\delta) t}\right)-\frac{1}{2} \frac{1-z_{0} \bar{z}_{0}}{1+z_{0} \bar{z}_{0}} \exp \left[-2\left(\eta+\delta+2 \frac{\sigma_{\xi}^{2}}{\nu_{\xi}+\omega_{0}^{2} / \nu_{\xi}}\right) t\right], \\
& W_{1}(t)=\frac{1}{2}+\frac{1}{2} \frac{\eta-\delta}{\eta+\delta}\left(1-\mathrm{e}^{-2(\eta-\delta) t}\right)+\frac{1}{2} \frac{1-z_{0} \bar{z}_{0}}{1+z_{0} \bar{z}_{0}} \exp \left[-2\left(\eta+\delta+2 \frac{\sigma_{\xi}^{2}}{\nu_{\xi}+\omega_{0}^{2} / \nu_{\xi}}\right) t\right] .
\end{aligned}
$$


As a result, we get formulas for longitudinal $T_{1}$ and transversal $T_{2}$ relaxation times:

$$
T_{1}=\frac{1}{2\left(\eta+\delta+2 \frac{\sigma_{\xi}^{2}}{\nu_{\xi}+\omega_{0}^{2} / \nu_{\xi}}\right)}, \quad T_{2}=\frac{1}{\eta+\delta+\frac{\sigma_{\Omega}^{2}}{\nu_{\Omega}}+2 \frac{\sigma_{\xi}^{2}}{\nu_{\xi}+\omega_{0}^{2} / \nu_{\xi}}},
$$

which are connected as follows:

$$
\frac{1}{2 T_{1}}+\frac{\sigma_{\Omega}^{2}}{\nu_{\Omega}}=\frac{1}{T_{2}}
$$

In the absence of random fields we get the well-known relation $T_{2}=2 T_{1}$.

\section{CONCLUSION}

In this paper we have considered briefly the problem of generation and destruction of atomic coherent states, which is very important for quantum calculations. Details of calculations and comparisons with different approaches (see, for example, paper [9]) will be published elsewhere.

Acknowledgements. The author would like to express his gratitude to Profs. S. I. Vinitsky and V.L. Derbov and Dr. V. A. Mikhailov for helpful discussion, and to the organizers of the Workshop for the kind invitation.

\section{REFERENCES}

1. Perelomov A. M. Generalized Coherent States and Their Applications. Berlin: Springer, 1986.

2. Gorokhov A. V. Group Theory Methods in Problems of Quantum Physics. Kuibyshev (Samara) State University. Kuibyshev, 1977; 1979; 1983. Parts 1-3 (in Russian).

3. Zhang W. M., Feng D. H., Gilmore R. // Rev. Mod. Phys. 1990. V.62. P. 867-927.

4. Gorokhov A. V., Mikhailov V. A. // Izv. vuzov. (Fizika). 1985. V.7. P. 59-64.

5. Gorokhov A. V., Mikhailov V.A. Quantum Kinetics of System Interacting with Heat Bath and Stochastic Field // Symmetry Methods in Physics. Obninsk, 1992. P. 120.

6. Gorokhov A. V., Mikhailov V.A. Fokker-Planck Equations in Coherent States Representation for Quantum Relaxation // Quantum Field Theory, Quantum Mechanics and Quantum Optics. N.Y., 1991. P. 233-235.

7. Stratonovich R. L. Topics in the Theory of Random Noise. N. Y.: Gordon and Breach, 1963.

8. Gorokhov A. V., Mikhailov V. A. // Theor. Phys. 2000. V.1. P. 54-62 (in Russian).

9. Petrov E. G., Goychuk I. A., May V. // Phys. Rev. E. 1996. V.54. P. 4500-4503. 